\title{
Analysis of Covariance of Reinforced Balanced Incomplete Block Designs With a Single Explanatory Variable
}

\author{
D. K. Ghosh \\ UGC BSR Faculty Fellow, Department of Statistics, Saurashtra University \\ Rajkot, Gujarat, India \\ ghosh_dkg@rediffmail.com \\ M. G. Bhatt \\ Department of Statistics, $H \&$ H.B. Kotak Institute of Science \\ Rajkot, Gujarat, India \\ mahendra.statistics@gmail.com \\ and \\ S. C. Bagui $^{\dagger}$ \\ Department of Mathematics and Statistics, The University of West Florida \\ Pensacola, FL 32514, USA \\ sbagui@uwf.edu \\ Received 5 March 2014 \\ Accepted 1 August 2014
}

Reinforced balanced incomplete block designs (BIBDs) are very useful in statistical planning of experiments as they can be constructed for any number of treatments for given numbers of replications. Das (1958) was first to introduce the statistical analysis of variance (ANOVA) of these designs, and in the same year Giri also developed the same statistical analysis of variance for reinforced partially balanced incomplete block designs (PBIBDs). In this article, we focus on the method of statistical analysis of covariance (ANCOVA) of reinforced balanced incomplete block design (BIBD) when a single explanatory variable is available in the experiment.

Keywords: Augmented designs, Reinforced designs, Reinforced BIBD, Reinforced PBIBD, and Ancillary variable.

AMS (2013) Mathematics Subject Classification: 62J, 62k

${ }^{\dagger}$ The corresponding author

Published by Atlantis Press

Copyright: the authors 


\section{Introduction}

The analysis of covariance (ANCOVA) is typically used to adjust or control for differences between treatment effects based another continuous variable called explanatory (or extraneous, or ancillary) variable (or covariate). In analysis of variance (ANOVA) if one or more explanatory variables are suspected to affect the outcome (dependent variable), then ignoring explanatory variables in ANOVA will increase error sum of squares and will make it harder to detect any real differences in treatment effects. Whereas the analysis covariance in this case will be more precise by reducing error sum of squares and increasing the statistical power of the experiment when a good covariate has been used within the ANCOVA. Keeping these in mind we will develop ANCOVA for reinforced BIBD,

Reinforced BIBD has several advantages over other designs particularly with respect to using any number of treatments for given numbers of replications. Thus it brings no limitation on statistical planning of experiments in different fields. In factorial experiments when the number of treatment combinations are large, the confounding techniques are used to reduce the block size. This procedure provides most accurate estimation of lower order interactions at the cost of some of the less important higherorder interactions which are confounded with blocks. Similar methods can be adopted for incomplete block designs in unifactorial situations such as one described below for the purpose of increasing precision.

If the number of replications of all pairs of treatment in a design are the same, it creates an important class of designs known as balanced incomplete block designs (BIBD). This classes of designs provide equal precisions of all estimates of all pairs of treatment effects. This was introduced by Yates in 1936 for agricultural experiments. It is known that the balanced incomplete block design is not always suitable for varietal trials since it requires large number of replications. In order to make incomplete block designs available for all treatments with smaller number of replications, Das (1958) and Giri (1958) introduced incomplete block designs augmented by certain number of additional treatments in each block. These new designs are called reinforced BIBD.

Suppose we have a BIBD with $v$ treatments, $b$ blocks of size $k$ each, and $r$ replications. We obtain reinforced balanced incomplete block designs by adding new treatments, say $p$ new ones, in each block to this BIBD. Then the resulting reinforced balanced incomplete block designs will have $(v+p)$ treatments distributed in $b$ blocks each of size $(k+p)$ such that each of $p$ newly introduced treatments are replicated $b$ times and the original $v$ treatments are replicated $r$ times each. In reinforced BIBD it is possible to introduce appropriate covariates in order to increase the precision in the design. In this article, we develop an exact method of analysis of covariance of reinforced balanced incomplete block designs with a single explanatory variable.

Summary of the results obtained in this paper are as follows. In section 2, we describe the model and notations for reinforced balanced incomplete block designs with one explanatory variable. The estimates of the model parameters are provided in section 3 . In section 4, we consider a model for $y$-variate without the explanatory variable and obtained least square (LS) estimates for parameters of this model. In this same section we 
define an ANOVA model for $x$-variate (the explanatory variable) only, and obtained LS estimates for the parameters. Actually this facilitates the calculations for the original model. Error sum of squares and adjusted treatment sum of squares, error sum of products and adjusted treatment sum of products for the above modified models are provided in section 4.1 and section 4.2, respectively. In section 5 we consider the model in presence of the explanatory variable. In this section, we provided error sum of squares and adjusted treatment sum of squares, error sum of products and adjusted treatment sum of products in presence of the explanatory $X$-variate.

Finally, variances of the estimable treatment contrast are obtained in section 6 . We present three types of variances, e.g., (i) $\operatorname{Var}\left(\hat{t}_{i}-\hat{t}_{j}\right)$, (ii) $\operatorname{Var}\left(\hat{t}_{i}-\hat{t}_{m}\right)$, and (iii) $\operatorname{Var}$ $\left(\hat{t}_{m}-\hat{t}_{m^{\prime}}\right)$, where $\hat{t}_{i}$ and $\hat{t}_{j}$ are the estimates of original treatment effects; $\hat{t}_{m}$ and $\hat{t}_{m^{\prime \prime}}$ are the estimates of the newly introduced treatment effects.

\section{Model and Notations}

Let $n_{i j}(i=1,2, \cdots, v+p ; j=1,2, \cdots, b)$ denote the number of times the $i$ th treatment occurs in $j$ th block and $n_{m j}(m=v+1, v+2, \cdots, v+p ; j=1,2, \cdots, b)$ denote the number of times $m$ th newly introduced treatment occurs in $j$ th block. In fact,

$$
n_{i j}=\left\{\begin{array}{l}
1 \text { if treatment } i \text { appears in block } j \\
0 \text { otherwise. }
\end{array}\right.
$$

In the context of reinforced BIBD, we have the following relationships:

$$
\begin{aligned}
& n_{. j}=\sum_{i=1}^{v+p} n_{i j}=\sum_{i=1}^{v} n_{i j}+\sum_{i=v+1}^{p} n_{i j}=(k+p), n_{. .}=\sum_{j=1}^{b} n_{. j}=b(k+p), \\
& n_{i .}=\sum_{j=1}^{b} n_{i j}=\left\{\begin{array}{l}
r \text { for } i=1,2, \cdots, v \\
b \text { for } i=v+1, \cdots, v+p
\end{array}, \sum_{j=1}^{b} n_{i j} n_{i^{\prime} j}=\lambda \text { for } i \neq i^{\prime}=1,2, \cdots, v,\right. \\
& \sum_{j=1}^{b} n_{i j} n_{m j}=r \text { for } i=1,2, \cdots, v ; m=v+1, \cdots, v+p, \text { and } \\
& \sum_{j=1}^{b} n_{m j} n_{m^{\prime} j}=b \text { for } m \neq m^{\prime}=v+1, \cdots, v+p .
\end{aligned}
$$

Suppose that $y_{i j}(i=1,2, \cdots, v+p ; j=1,2, \cdots, b)$ are the responses from the unit in $j$ th block with $i$ th treatment. The linear additive ANCOVA model for reinforced balanced incomplete block design is given by

$$
y_{i j}=\mu+t_{i}+b_{j}+\beta x_{i j}+e_{i j}
$$


where $\mu$ is a constant mean effect; $t_{i}$ is the effect of $i$ th treatment; $b_{j}$ is the effect of $j$ th block; $x_{i j}$ denote the explanatory variable from the unit in $j$ th block with $i$ th treatment; $e_{i j}$ are the error components that are assumed to be normally distributed with a zero mean and a constant variance $\sigma^{2} ; \beta$ is the regression coefficient.

\section{Estimation of Parameters with a Single Explanatory Variable}

The estimate of the unknown parameters $\mu, t_{i}, b_{j}$, and $\beta$ are obtained by minimizing the error sum of squares, $E$, where $E$ is given by

$$
E=\sum_{i=1}^{v+p} \sum_{j=1}^{b} n_{i j}\left(y_{i j}-\mu-t_{i}-b_{j}-\beta x_{i j}\right)^{2}
$$

The equation (3.1) provides the following normal equations,

$$
\begin{gathered}
G=b(k+p) \hat{\mu}+r \sum_{i=1}^{v} \hat{t}_{i}+b \sum_{m=v+1}^{v+p} \hat{t}_{m}+(k+p) \sum_{j=1}^{b} \hat{b}_{j}+\hat{\beta} G_{x} \\
B_{j}=(k+p) \hat{\mu}+\sum_{i=1}^{v} n_{i j} \hat{t}_{i}+\sum_{m=v+1}^{v+p} n_{m j} \hat{t}_{m}+(k+p) \hat{b}_{j}+\hat{\beta} B_{j(x)} \\
T_{i}=r \hat{\mu}+r \hat{t}_{i}+\sum_{j=1}^{b} n_{i j} \hat{b}_{j}+\hat{\beta} T_{i(x)}, \quad \forall i=1,2, \cdots, v \\
T_{m}=b \hat{\mu}+b \hat{t}_{m}+\sum_{j=1}^{b} n_{i j} \hat{b}_{j}+\hat{\beta} T_{m(x)}, \forall m=v+1, \cdots, v+p \\
\sum_{i=1}^{v+p} \sum_{j=1}^{b} n_{i j} x_{i j} y_{i j}=\hat{\mu} G_{x}+\sum_{i=1}^{v} \hat{t}_{i} T_{i(x)}+\sum_{m=v+1}^{v+p} \hat{t}_{m} T_{m(x)}+\sum_{j=1}^{b} \hat{b}_{j} B_{j(x)}+\hat{\beta} \sum_{i=1}^{v+p} \sum_{j=1}^{b} n_{i j} x_{i j}^{2}
\end{gathered}
$$

where $G$ is the grand total; $G_{x}$ is the grand total for the $x$-variate; $B_{j}$ is the $j$ th block total; $B_{j(x)}$ is the $j$ th block total for the $x$-Variate; $T_{i}$ and $T_{m}$ are the treatment totals for $v$ original treatments and $p$ newly introduced treatments, respectively. Below we give their mathematical expressions.

$$
\begin{gathered}
G=\sum_{i=1}^{v+p} \sum_{j=1}^{b} n_{i j} y_{i j}, G_{x}=\sum_{i=1}^{v+p} \sum_{j=1}^{b} n_{i j} x_{i j}, B_{j}=\sum_{i=1}^{v+p} n_{i j} y_{i j}, B_{j(x)}=\sum_{i=1}^{v+p} n_{i j} x_{i j} ; \\
T_{i}=\sum_{j=1}^{b} n_{i j} y_{i j}, T_{i(x)}=\sum_{j=1}^{b} n_{i j} x_{i j}, \forall i=1,2, \cdots, v ; T_{m}=\sum_{j=1}^{b} n_{m j} y_{m j} \text { and } T_{m(x)}=\sum_{j=1}^{b} n_{m j} x_{m j}, \\
\forall m=v+1, \cdots, v+p .
\end{gathered}
$$


Now applying the restrictions, $\sum_{j=1}^{b} \hat{b}_{j}=0$ and $r \sum_{i=1}^{v} \hat{t}_{i}+b \sum_{m=v+1}^{v+p} \hat{t}_{m}=0$ in the above normal equations, the expressions for the estimates of $\mu$ and $b_{j}$ are given by

$$
\begin{gathered}
\hat{\mu}=\frac{1}{b(k+p)}\left(G-\hat{\beta} G_{x}\right) \\
\hat{b}_{j}=\frac{1}{(k+p)}\left[B_{j}-(k+p) \hat{\mu}-\sum_{i=1}^{v} n_{i j} \hat{t}_{i}-\sum_{m=v+1}^{v+p} n_{m j} \hat{t}_{m}-\hat{\beta} B_{j(x)}\right]
\end{gathered}
$$

where the estimate of $t_{i}$ and $t_{m}$ are obtained in this section below and the estimate $\hat{\beta}$ is provided in section 5. Now substituting $\hat{b}_{j}$ in (3.5), and after simplifications we have

$$
Q_{m}=b \hat{t}_{m}-\frac{b}{(k+p)} \sum_{m^{\prime}=v+1}^{v+p} \hat{t}_{m^{\prime}}-\frac{r}{(k+p)} \sum_{i^{\prime}=1}^{v} \hat{t}_{i^{\prime}}+\hat{\beta} Q_{m(x)}
$$

where $Q_{m}$ is the adjusted total for $m$ th treatment and $Q_{m(x)}$ is the adjusted total for $m$ th treatment for $x$-variate. The expressions for $Q_{m}$ and $Q_{m(x)}$ are given below:

$$
\begin{gathered}
Q_{m}=T_{m}-\frac{1}{(k+p)} \sum_{j=1}^{b} n_{m j} B_{j} \text { and } Q_{m(x)}=T_{m(x)}-\frac{1}{(k+p)} \sum_{j=1}^{b} n_{m j} B_{j(x)}, \\
\forall m=v+1, \cdots, v+p
\end{gathered}
$$

Now the restriction $r \sum_{i^{\prime}=1}^{v} \hat{t}_{i^{\prime}}+b \sum_{m^{\prime}=v+1}^{v+p} \hat{t}_{m^{\prime}}=0$ reduces (3.9) to

$$
Q_{m}=b \hat{t}_{m}+\hat{\beta} Q_{m(x)}
$$

Thus, the estimate of $t_{m}$ may be expressed as

$$
\hat{t}_{m}=\frac{1}{b}\left(Q_{m}-\hat{\beta} Q_{m(x)}\right), \forall m=v+1, \cdots, v+p
$$

Also, using (3.11) we may write

$$
\sum_{m^{\prime}=v+1}^{v+p} \hat{t}_{m^{\prime}}=\frac{1}{b}\left(\sum_{m^{\prime}=v+1}^{v+p} Q_{m^{\prime}}-\hat{\beta} \sum_{m^{\prime}=v+1}^{v+p} Q_{m^{\prime}(x)}\right) .
$$

Similarly, the estimate of $i$ th treatment effect $t_{i}$ is obtained by substituting $\hat{b}_{j}$ from (3.8) in (3.4) and applying (3.12). Therefore, after more simplifications $\hat{t}_{i}$ is given by

$$
\hat{t}_{i}=\frac{1}{z}\left[(k+p) Q_{i}+\frac{1}{b}\left(r-\frac{\lambda b}{r}\right)\left(\sum_{m^{\prime}=v+1}^{v+p} Q_{m^{\prime}}-\hat{\beta} \sum_{m^{\prime}=v+1}^{v+p} Q_{m^{\prime}(x)}\right)-\hat{\beta}(k+p) Q_{i(x)}\right]
$$


where $z=r(k+p)-r+\lambda ; \hat{t}_{i}$ stand for estimate of the original effects, $i=1,2, \cdots, v$; $\hat{t}_{m}$ stand for estimate of the newly introduced treatment effects, $m=v+1, \cdots, v+p ; Q_{i}$ and $Q_{i(x)}$ are given, respectively, by

$$
Q_{i}=T_{i}-\frac{1}{(k+p)} \sum_{j=1}^{b} n_{i j} B_{j} \text { and } Q_{i(x)}=T_{i(x)}-\frac{1}{(k+p)} \sum_{j=1}^{b} n_{i j} B_{j(x)} .
$$

\section{Estimation of Parameters without an Explanatory Variable}

The model (2.1) without the explanatory $x$-variate can be written as

$$
y_{i j}=\mu_{y}+t_{i(y)}+b_{j(y)}+e_{i j(y)}
$$

where the notations $\mu_{y}, t_{i(y)}, b_{j(y)}$, and $e_{i j(y)}$ have similar meaning and satisfy similar assumptions as discussed in section 2. Now using least square method of estimation as before, we obtain the estimates of the parameters as follows.

$$
\begin{gathered}
\hat{\mu}_{y}=\frac{G}{b(k+p)} \\
\hat{b}_{j(y)}=\frac{1}{(k+p)}\left[B_{j}-(k+p) \hat{\mu}_{y}-\sum_{i=1}^{v} n_{i j} \hat{t}_{i(y)}-\sum_{m=v+1}^{v+p} n_{m j} \hat{t}_{m(y)}\right] \\
\hat{t}_{i(y)}=\frac{1}{[r(k+p)-r+\lambda]}\left[(k+p) Q_{i}+\frac{1}{b}\left(r-\frac{\lambda b}{r}\right) \sum_{m^{\prime}=v+1}^{v+p} Q_{m^{\prime}}\right], \forall i=1,2, \cdots, v \\
\hat{t}_{m(y)}=\frac{Q_{m}}{b}, \forall m=v+1, \cdots, v+p .
\end{gathered}
$$

Now replacing $y_{i j}$ in (4.1) by $x_{i j}$, we get the following new model. The model in terms of $x$-variate may be written as

$$
x_{i j}=\mu_{x}+t_{i(x)}+b_{j(x)}+e_{i j(x)}
$$

where $\mu_{x}, t_{i(x)}, b_{j(x)}$, and $e_{i j(x)}$ have similar meaning and satisfy similar assumptions as discussed in section 2 with respect to the explanatory $x$-variate. Now using least square method of estimation as before, we obtain the estimates of the parameters given (4.6) as

$$
\begin{gathered}
\hat{\mu}_{x}=\frac{G_{x}}{b(k+p)} \\
\hat{b}_{j(\mathrm{x})}=\frac{1}{(k+p)}\left[B_{j(x)}-(k+p) \hat{\mu}_{x}-\sum_{i=1}^{v} n_{i j} \hat{t}_{i(x)}-\sum_{m=v+1}^{v+p} n_{m j} \hat{t}_{m(x)}\right]
\end{gathered}
$$




$$
\begin{gathered}
\hat{t}_{i(x)}=\frac{1}{[r(k+p)-r+\lambda]}\left[(k+p) Q_{i(x)}+\frac{1}{b}\left(r-\frac{\lambda b}{r}\right) \sum_{m^{\prime}=v+1}^{v+p} Q_{m^{\prime}(x)}\right], \forall i=1,2, \cdots, v \\
\hat{t}_{m(x)}=\frac{Q_{m(x)}}{b}, \quad \forall m=v+1, \cdots, v+p .
\end{gathered}
$$

The estimates given in equations (4.2)-(4.5) and (4.7)-(4.10) are necessary to compute the expressions $E_{y y}$ and $E_{x y}$ given in section 4.1 and 4.2 respectively. In turn these will help in calculating $\hat{\beta}$, the regression coefficient, given in section 5 below.

\subsection{Error Sum of Squares and Adjusted Treatment Sum of Squares for $y$-variate with no Explanatory Variable}

The error sum of squares for the model (4.1) can be written as

$$
E_{y y}=\sum_{i=1}^{v+p} \sum_{j=1}^{b} n_{i j} y_{i j}^{2}-\mu_{y} G-\sum_{i=1}^{v} t_{i(y)} T_{i}-\sum_{m=v+1}^{v+p} t_{m(y)} T_{m}-\sum_{j=1}^{b} b_{j(y)} B_{j}
$$

Now substituting $\hat{\mu}_{y}$ and $\hat{b}_{j(y)}$ from (4.2) and (4.3), receptively, in the above equation, we obtain

$$
E_{y y}=\sum_{i=1}^{v+p} \sum_{j=1}^{b} n_{i j} y_{i j}^{2}-\frac{1}{(k+p)} \sum_{j=1}^{b} B_{j}^{2}-\left(\sum_{i=1}^{v} t_{i(y)} Q_{i}+\sum_{m=v+1}^{v+p} t_{m(y)} Q_{m}\right)
$$

Under the null hypothesis $H_{o}: t_{i(y)}=0, \forall i=1,2, \cdots, v$ and $t_{m(y)}=0$, $\forall m=v+1, \cdots, v+p$ the above equation (4.1.1) reduces to

$$
E_{y y}^{\prime}=\sum_{i=1}^{v+p} \sum_{j=1}^{b} n_{i j} y_{i j}^{2}-\frac{1}{(k+p)} \sum_{j=1}^{b} B_{j}^{2} .
$$

Thus the adjusted treatment sum of squares for $y$-variate on the assumption of no explanatory variable is given by

$$
E_{y y}=E_{y y}^{\prime}-E_{y y}=\left(\sum_{i=1}^{v} \hat{t}_{i(y)} Q_{i}+\sum_{m=v+1}^{v+p} \hat{t}_{m(y)} Q_{m}\right)
$$

Similarly, using model (4.6) the quantities $E_{x x}$, the error sum of squares for $x$-variate and $E_{x x}^{\prime}$, the error sum of squares for $x$-variate under $H_{o}: t_{i(x)}=0, \forall i=1,2, \cdots, v$ and $t_{m(x)}=0, \forall m=v+1, \cdots, v+p$, and $T_{x x}$, adjusted treatment sum of squares for $x$ variate, can easily be obtained. In the similar manner the expression for $T_{x x}$ will be 


$$
T_{x x}=E_{x x}^{\prime}-E_{x x}=\left(\sum_{i=1}^{v} \hat{t}_{i(x)} Q_{i(x)}+\sum_{m=v+1}^{v+p} \hat{t}_{m(x)} Q_{m(x)}\right) .
$$

\subsection{Error Sum of Products and Adjusted Treatment Sum of Products}

The error sum of products of $x$ and $y$ can be obtained using models (4.1) and (4.6) as

$$
E_{x y}=\sum_{i=1}^{v+p} \sum_{j=1}^{b} n_{i j} x_{i j} y_{i j}-\mu_{x} G-\sum_{i=1}^{v} t_{i(x)} T_{i}-\sum_{m=v+1}^{v+p} t_{m(x)} T_{m}-\sum_{j=1}^{b} b_{j(x)} B_{j}
$$

Now substituting $\hat{\mu}_{x}$ and $\hat{b}_{j(x)}$ from (4.7) and (4.8), respectively, in the above equation (4.2.1), we get

$$
E_{x y}=\sum_{i=1}^{v+p} \sum_{j=1}^{b} n_{i j} x_{i j} y_{i j}-\frac{1}{(k+p)} \sum_{j=1}^{b} B_{j} B_{j(x)}-\left(\sum_{i=1}^{v} t_{i(x)} Q_{i(x)}+\sum_{m=v+1}^{v+p} t_{m(x)} Q_{m(x)}\right)
$$

Under the hypothesis $H_{o}: t_{i(x)}=0, \forall i=1,2, \cdots, v$ and $t_{m(x)}=0, \forall m=v+1, \cdots, v+p$ the above equation (4.2.2) reduces to

$$
E_{x y}^{\prime}=\sum_{i=1}^{v+p} \sum_{j=1}^{b} n_{i j} x_{i j} y_{i j}-\frac{1}{(k+p)} \sum_{j=1}^{b} B_{j} B_{j(x)}
$$

Thus the expression for adjusted treatment sum of products is given by

$$
T_{x y}=E_{x y}^{\prime}-E_{x y}=\left(\sum_{i=1}^{v} \hat{t}_{i(x)} Q_{i}+\sum_{m=v+1}^{v+p} \hat{t}_{m(x)} Q_{m}\right) .
$$

\section{Error Sum of Squares and Adjusted Treatment Sum of Squares for $y$-variate in Presence of an Explanatory $x$-variate}

In this section, we will focus on obtaining estimate of regression coefficient, error sum of squares, and adjusted treatment sum of squares for $y$-variate in presence of ancillary $x$ variate. Below we state a useful lemma. This lemma helps to derive the estimates of error sum of squares, adjusted treatment sum of squares, and regression coefficient $(\beta)$.

Lemma 5.1. For analysis of covariance of reinforced BIBD in presence of a single explanatory variable the parameters $\mu_{y}, t_{i(y)}, t_{m(y)}$, and $b_{j(y)}$ are estimated as

$$
\hat{\mu}_{y}=\hat{\mu}+\hat{\beta} \hat{\mu}_{x} ; \hat{t}_{i(y)}=\hat{t}_{i}+\hat{\beta} \hat{t}_{i(x)} ; \hat{t}_{m(y)}=\hat{t}_{m}+\hat{\beta} \hat{t}_{m(x)} ; \hat{t}_{j(y)}=\hat{b}_{j}+\hat{\beta} \hat{b}_{j(x)} .
$$

Proof. Substituting the values of $x_{i j}$ from (4.6) in (2.1), we have

$$
y_{i j}=\left(\mu+\beta \mu_{x}\right)+\left(t_{i}+\beta t_{i(x)}\right)+\left(t_{m}+\beta t_{m(x)}\right)+\left(b_{j}+\beta b_{j(x)}\right)+\left(e_{i j}+\beta e_{i j(x)}\right)
$$


Now, on comparing the coefficients of (5.1) with (4.1) we obtain

$$
\hat{\mu}_{y}=\hat{\mu}+\hat{\beta} \hat{\mu}_{x} ; \hat{t}_{i(y)}=\hat{t}_{i}+\hat{\beta} \hat{t}_{i(x)} ; \hat{t}_{m(y)}=\hat{t}_{m}+\hat{\beta} \hat{t}_{m(x)} ; \hat{t}_{j(y)}=\hat{b}_{j}+\hat{\beta} \hat{b}_{j(x)} .
$$

This completes the proof.

\subsection{Estimate of Regression Coefficient $\beta$}

We get the estimate of $\beta$ from the normal equation (3.6). Now on substituting $\hat{\mu}$ and $\hat{b}_{j}$ from (3.7) and (3.8), respectively, $\hat{t}_{i}=\hat{t}_{i(y)}-\beta \hat{t}_{i(x)}$, and $\hat{t}_{m}=\hat{t}_{m(y)}-\beta \hat{t}_{m(x)}$ in (3.6), we obtain $E_{x y}=\hat{\beta} E_{x x}$. Final we have

$$
\hat{\beta}=\frac{E_{x y}}{E_{x x}}
$$

Under the hypothesis $H_{o}: t_{i(x)}=0, \forall i=1,2, \cdots, v$ and $t_{m(x)}=0, \forall m=v+1, \cdots, v+p$ (5.1.1) will reduce to

$$
\beta^{\prime}=\frac{E_{x y}^{\prime}}{E_{x x}^{\prime}}
$$

\subsection{Error sum of squares and adjusted Treatment Sum of Squares}

From (2.1), the error sum of squares of $y$-variate with an explanatory variable $x$ can be found as

$$
E=\sum_{i=1}^{v+p} \sum_{j=1}^{b} n_{i j} y_{i j}^{2}-\mu G-\sum_{i=1}^{v} t_{i} T_{i}-\sum_{m=v+1}^{v+p} t_{m} T_{m}-\sum_{j=1}^{b} b_{j} B_{j}-\beta \sum_{i=1}^{v+p} \sum_{j=1}^{b} n_{i j} x_{i j} y_{i j} .
$$

On substituting $\hat{\mu}$ and $\hat{b}_{j}$ from (3.7) and (3.8), respectively, $\hat{t}_{i}=\hat{t}_{i(y)}-\beta \hat{t}_{i(x)}$, and $\hat{t}_{m}=$ $\hat{t}_{m(y)}-\beta \hat{t}_{m(x)}$ in (5.2.1) we get

$$
E=E_{y y}-\hat{\beta} E_{x y}
$$

Under the hypothesis $H_{o}: t_{i(x)}=0, \forall i=1,2, \cdots, v$ and $t_{m(x)}=0, \forall m=v+1, \cdots, v+p$ (5.2.2) will reduce to

$$
E_{1}=E_{y y}^{\prime}-\hat{\beta}^{\prime} E_{x y}
$$

Thus the adjusted treatment sum of squares of $y$-variate given the explanatory $x$-variate is obtained as the difference $\left(E_{1}-E\right)$. The above results are summarized in the form of 
Table 1 and Table 2. Table 1 shows sums of squares and sums of products and Table 2 shows adjusted error and treatment sums of squares.

Table 1. Variance-Covariance Table for Analysis of Covariance of Reinforced BIBD with a Single Explanatory Variable

\begin{tabular}{|l|l|l|l|l|}
\hline Source & d.f. & $x^{2}$ & $x y$ & $y^{2}$ \\
\hline Block(unadj.) & $b-1$ & $B_{x x}$ & $B_{x y}$ & $B_{y y}$ \\
\hline Treatment(adj.) & $v+p-2$ & $T_{x x}$ & $T_{x y}$ & $T_{y y}$ \\
\hline Error & $v r+p b-v-p-b+2$ & $E_{x x}$ & $E_{x y}$ & $E_{y y}$ \\
\hline Total & $v r+p b-1$ & $S_{x x}$ & $S_{x y}$ & $S_{y y}$ \\
\hline
\end{tabular}

Table 2. Adjusted Sums of Squares

\begin{tabular}{|c|c|c|c|c|c|c|}
\hline Source & d.f. & $x^{2}$ & $x y$ & $y^{2}$ & $\beta$ & Adj. S.S. \\
\hline Treat(Adj.) & $v+p-2$ & $T_{x x}$ & $T_{x y}$ & $T_{y y}$ & & $E_{1}-E$ \\
\hline Error & $v r+p b-v-p-b+1$ & $E_{x x}$ & $E_{x y}$ & $E_{y y}$ & $\hat{\beta}=E_{x y} / E_{x x}$ & $E=E_{y y}-\hat{\beta} E_{x y}$ \\
\hline Treat+Error & $v r+p b-b-1$ & $E_{x x}^{\prime}$ & $E_{x y}^{\prime}$ & $E_{y y}^{\prime}$ & $\hat{\beta}^{\prime}=E_{x y}^{\prime} / E_{x x}$ & $E_{1}=E_{y y}^{\prime}-\hat{\beta}^{\prime} E_{x y}^{\prime}$ \\
\hline
\end{tabular}

Now the appropriate test statistics for the hypothesis $H_{o}: t_{i(x)}=0, \forall i=1,2, \cdots, v$ and $t_{m(x)}=0, \forall m=v+1, \cdots, v+p$ and is given by

$$
F=\frac{\left(E_{1}-E\right) / f_{1}}{E / f_{2}}
$$

where $F$ follows an distribution with $f_{1}=v+p-2$ and $f_{2}=v r+p b-v-p-b+1$. The null hypothesis $H_{o}$ is rejected if $F>F_{\alpha ; f_{1}, f_{2}}$ where $F_{\alpha ; f_{1}, f_{2}}$ is an upper $\alpha$ critical point of distribution with $f_{1}$ and $f_{2}$ degrees of freedom.

\section{Variance of Treatment Contrasts}

Three types of treatment contrasts can be considered for this type of designs. Variances of these contrasts are described as follows.

Case-I. The estimated variance of the estimable treatment contrast $\left(\hat{t}_{i}-\hat{t}_{i^{\prime}}\right)$, $i \neq i^{\prime}=1,2, \cdots, v \quad$ can be found using (3.13) as 


$$
\operatorname{Var}\left(\hat{t}_{i}-\hat{t}_{i^{\prime}}\right)=\left[\frac{2(k+p)}{r(k+p)-r+\lambda}+\frac{\left(Q_{i(x)}-Q_{i^{\prime}(x)}\right)^{2}}{E_{x x}}\right] \hat{\sigma}^{2} .
$$

Since the variance between two adjusted treatment estimates is different for each comparison, Finney (1946) and Das and Giri (1986) suggested that an average value of $\left(Q_{i(x)}-Q_{i^{\prime}(x)}\right)^{2}$ can be replaced by $T_{i(x)} /(v-1)$ and hence average variance of treatment contrast can be obtained as

$$
\text { 'average' } \operatorname{Var}\left(\hat{t}_{i}-\hat{t}_{i^{\prime}}\right)=\left[\frac{2(k+p)}{r(k+p)-r+\lambda}+\frac{T_{i(x)}}{(v-1) E_{x x}}\right] \hat{\sigma}^{2}
$$

where $\hat{\sigma}^{2}$ is the error mean squares. It can be obtained from Table 2 as $\hat{\sigma}^{2}=E /(v r+p b-v-p-b+1)$.

Case-II. The estimated variance of the estimable treatment contrast $\left(\hat{t}_{i}-\hat{t}_{m}\right)$, $i=1,2, \cdots, v ; m=v+1, \cdots, v+p$ can be found from (3.11) and (3.13) as

$$
\operatorname{Var}\left(\hat{t}_{i}-\hat{t}_{i^{\prime}}\right)=\left[\begin{array}{l}
\left\{\frac{(k+p)}{z}+\frac{r^{2}-\lambda b}{b r z}+\frac{1}{b}\right\}+ \\
\frac{1}{E_{x x}}\left\{\frac{1}{z}\left[\frac{1}{b}\left(r-\frac{\lambda b}{r}\right)\left(\sum_{m^{\prime}} Q_{m^{\prime}(x)}\right)+(k+p) Q_{i(x)}\right]-\frac{1}{b} \sum_{m^{\prime}} Q_{m^{\prime}(x)}\right\}
\end{array}\right] \hat{\sigma}^{2}
$$

where $z=r(k+p)-r+\lambda$.

Case-III. The estimated variance of the estimable treatment contrast $\left(\hat{t}_{m}-\hat{t}_{m^{\prime}}\right)$, $m \neq m^{\prime}=v+1, \cdots, v+p \quad$ can be found from (3.11) as

$$
\operatorname{Var}\left(\hat{t}_{m}-\hat{t}_{m^{\prime}}\right)=\frac{1}{b}\left[2+\frac{\left(Q_{m(x)}-Q_{m^{\prime}(x)}\right)^{2}}{E_{x x}}\right] \hat{\sigma}^{2} .
$$

The estimated average variance of treatment contrast $\left(\hat{t}_{m}-\hat{t}_{m^{\prime}}\right)$ can be found on substituting the average value of $\left(Q_{m(x)}-Q_{m^{\prime}(x)}\right)^{2}$ by $T_{m(x)} /(p-1)$ in (6.4) as 


$$
\text { 'average' } \operatorname{Var}\left(\hat{t}_{m}-\hat{t}_{m^{\prime}}\right)=\frac{1}{b}\left[2+\frac{T_{m(x)}}{(p-1) E_{x x}}\right] \hat{\sigma}^{2}
$$

where $\hat{\sigma}^{2}$ is obtained as before.

\section{Acknowledgements}

The authors are thankful to the Editor-in-Chief and an anonymous referee for their careful reading of the paper. Dr Bagui's research was partly supported by UWF faculty catalyst initiative award.

\section{References}

[1] M.N. Das, Reinforced incomplete block designs, Journal of the Indian Society of Agricultural Statistics, 10 (1958) 73-77.

[2] M.N. Das and N.C. Giri, Design and Analysis of Experiments, 2nd ed., (Wiley Eastern Ltd., New Delhi, 1986).

[3] D.J. Finney, Standard errors of yield adjusted for regression on an independent measurement, Biometrics, 2 (1946) 53-55.

[4] N.C. Giri, On reinforced PBIB designs, Jour. Ind. Agri. Stat., 12(1958) 41-51.

[5] F. Yates, Incomplete randomized blocks, Annals of Eugenics, 7(2) (1936) 121-140. 